\title{
FORCES ACTING ON THE SHIELDED THREE-PHASE SUPPLY
}

\author{
Daniel MAYER, Bohuš ULRYCH, Petr POLCAR \\ *Faculty of Electrical Engineering, University of West Bohemia, Univerzitni 26, Pilsen 30614, Czech Republic, tel. +420/37763 4601, \\ E-mail:\{mayer, ulrych, polcarp\}@kte.zcu.cz
}

\begin{abstract}
Mechanical forces impact three-phase line conducted by large currents. These forces cause higher safety requirements. This paper deals with possibilities of lowering these forces by shielding of the conductors. The numerical method of calculation of the time function of forces, when conductors are shielded by ferromagnetic jackets and conducted by symmetrical three-phase system currents, is stated. The calculation of eddy currents density induced into shielding jackets is stated further; eddy currents cause the heating of shielding jackets. An illustrative example shows that properly designed shielding is able to strongly reduce the magnitude of forces while the eddy current losses density is very low is the heating is subtle.
\end{abstract}

Keywords: Forces on three-phase line, magnetic shileding, eddy currents in ferromagnetic material, Joule losses density

\section{INTRODUCTION}

Power engineering devices working with shortage currents are exerted by high mechanical forces. This is met e.g. in heads of turbo-alternators winding, outlets of large rotating machines or power transformers, inlets of arc furnaces, etc. These forces can be restricted by shielding [1]. However, improperly made shielding can reduce the forces only insufficiently, or it can cause excessive eddy currents losses in shielding jacket followed by heating, or it can cause insufficient heat conduction by cooling medium.

This article contributes to the investigation of data required for proper construction design of shielding system. The method of investigation of time functions of forces acting on the copper conductive cables of threephase grounded system is described. The method is demonstrated with an numerical example computed with the help of software QuickField 5.0.

\section{FORMULATION OF THE PROBLEM}

Outlets of the three-phase alternator are made of triplet of straight, copper conductive cables of circular crosssection. Current leaders are placed in steel jackets enduring magnetic shielding (Fig. 1) and flowed by currents $i_{X}(t)$, $\left.i_{\mathrm{Z}}(t), i_{\mathrm{Z}}(t)\right)$ of symmetrical three-phase system $(\mathrm{X}, \mathrm{Y}, \mathrm{Z})$. We will investigate:

- Time function of forces $f_{\mathrm{X}}(t), f_{\mathrm{Z}}(t), f_{\mathrm{Z}}(t)$ acting on the current leaders, if the currents change in time harmonically:

$$
\begin{aligned}
& i_{\mathrm{X}}(t)=B \cos \omega t \\
& i_{\mathrm{Y}}(t)=B \cos (\omega t+2 \pi / 3) \\
& i_{\mathrm{Z}}(t)=B \cos (\omega t-2 \pi / 3)
\end{aligned}
$$

- Density of Joule losses of eddy currents in shielding jackets.

To evaluate the effectiveness of shielding the forces $f_{X}(t)$, $f_{\mathrm{Y}}(t), f_{\mathrm{Z}}(t)$ acting on the conductors, that are not shielded magnetically, will be investigated.

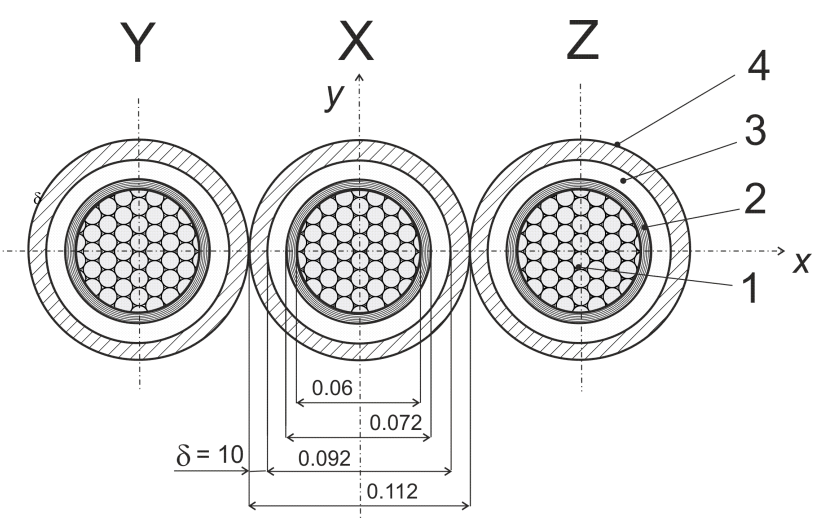

Fig. 1 Arrangement of the shielded conductors. Cross-sections of individual components with definition subareas. 1: copper conductive cable, 2: insulation, 3 : setting blocks, 4 : shielding jackets, 5: outer air.

\section{MATHEMATICAL MODEL}

Differential equations describing the electromagnetic field in the individual definition subareas 1 to 5 , equations of the forces acting on the conductors and furthermore densities of the Joule losses in shielding jackets will be stated. Generally, time-variant electromagnetic field in a non-linear ferromagnetic material is described by the equation

$\operatorname{curl} \frac{1}{\mu} \operatorname{curl} \mathbf{A}=-\gamma \frac{\partial \mathbf{A}}{\partial t}+\mathbf{J}_{\mathrm{ext}}$

In our case, this equation is valid: $\mathbf{J}_{\text {ext }}=\mathbf{z}_{0} J_{z \text {,ext }}(t)$ and $\mathbf{A}=\mathbf{z}_{0} A_{z}(x, y, t)$.

a) in the subarea of the cross-section of conductors $\mathbf{1}$ :

$\frac{\partial^{2} A_{z}}{\partial x^{2}}+\frac{\partial^{2} A_{z}}{\partial y^{2}}=\mu_{0} J_{z, \text { ext }}$

b) in the subarea of cross-sections on electric insulation 2, setting blocks $\mathbf{3}$ and outer air $\mathbf{5}$ :

$\frac{\partial^{2} A_{z}}{\partial x^{2}}+\frac{\partial^{2} A_{z}}{\partial y^{2}}=0$ 
c) in the subarea of cross sections of shielding jackets 4 :

$\frac{\partial}{\partial x} \frac{1}{\mu} \frac{\partial A_{z}}{\partial x}+\frac{\partial}{\partial y} \frac{1}{\mu} \frac{\partial A_{z}}{\partial y}=\gamma \frac{\partial A_{z}}{\partial t}$

Force acting on the conductor with current density $\mathbf{J}$ in the area of the magnetic field $\mathbf{B}=$ curl $\mathbf{A}$ can generally be expressed in the form

$\mathbf{F}=\int_{V}(\mathbf{J} \times \mathbf{B}) \mathrm{d} V$

where $V$ states the volume of the conductor. For the conductors according to Fig.1., for one meter of the length of the conductor with the cross-section $S$

$f_{x}=-J_{z, \text { ext }} \int_{S} B_{y} \mathrm{~d} S$

$f_{y}=-J_{z, \text { ext }} \int_{S} B_{x} \mathrm{~d} S$

Because the line is symmetrical to $x$ axis (Fig.1), the force acting on conductors has only $x$ component $f_{x}$, (the index will not be mentioned further), while $f_{y}=0$.

The average volume Joule losses density $w_{\mathrm{av}}(x, y, t)$ in shielding jackets is

$w_{\mathrm{av}}=\frac{1}{V} \int_{0}^{V} w(x, y, t) \mathrm{d} V$

and time average losses density is

$\bar{w}_{\mathrm{av}}=\frac{1}{T} \int_{0}^{T} w_{\mathrm{av}}(t) \mathrm{d} t$

where $V$ states the volume of the shielding jacket, $T$ is the time of one period of forces, $x, y \in V ; t \in T$.

\section{NUMERICAL MODEL}

Numerical solution of the mathematical model stated in the paragraph 3 was solved with the use of FEM by software QuickField 5.0, [3], in module TransientElectromagnetics. The convergence of numerical solution was observed - the influence of the discretization $\delta_{\min }, \delta_{\max }$ of geometrical definition area as the influence of the time discretization $\Delta t$ was observed. It was showed, that the accuracy of results - forces acting on the conductors - is affected especially by the values $\delta_{\min }$ (the discretization of cross-sections of conductors and shielding components. The discretization $\delta_{\max }$ of the outer air practically does not influence the accuracy of the solution. It was further proved, that the influence of the discretization $\Delta t$ compared to the influence of $\delta_{\text {min }}$ is less substantial. It is apparently related to the second derivatives by geometrical variables and the second derivatives by time in solved differential equations. It can be stated, that maximal values of the forces among conductors can be determined with the accuracy of 3 valid numbers by values $\delta_{\min }=0.001 \mathrm{~m}, \delta_{\max }=0.025 \mathrm{~m}$.

Lines of force of the magnetic field $A_{z}(x, y, t)=$ const. are stated for the illustration of obtained results for these examples:
- conductors are not shielded, Fig. 2,

- conductors are shielded by steel shielding jacket of $\delta=10 \mathrm{~mm}$, Fig. 3, thickness $\delta=5 \mathrm{~mm}$, Fig. 4 .

Input data of cases presented in these figures are stated in paragraph 5.1, discussion of the results is stated in paragraphs 5.2.1. and 5.2.2.

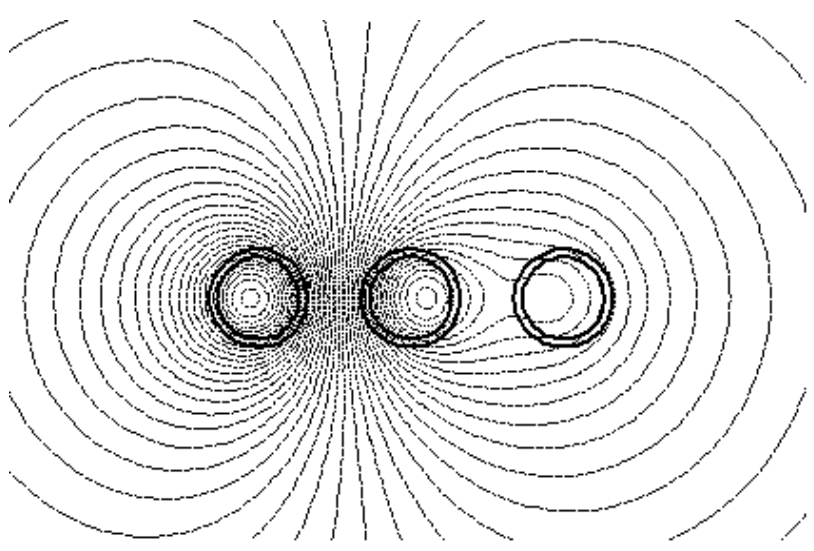

Fig. 2 Magnetic field lines of force of unshielded conductors.

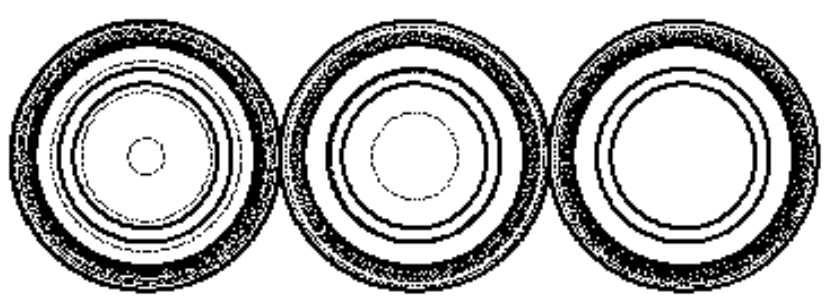

Fig. 3 Magnetic field lines of force of shielded conductors $(\delta=10 \mathrm{~mm})$.

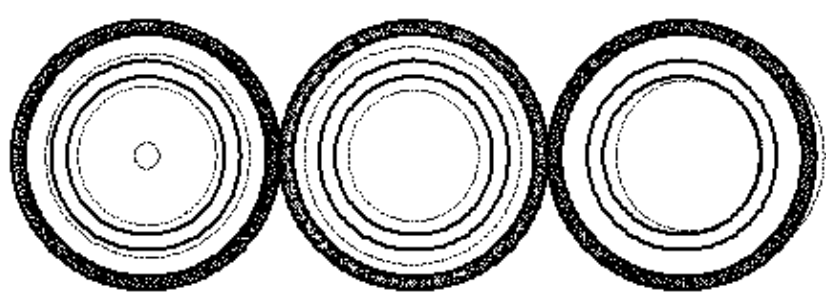

Fig. 4 Magnetic field lines of force of shielded conductors $(\delta=5 \mathrm{~mm})$.

By comparing the Fig. 2 with Figs. 3 and 4 it is obvious, that magnetic field acting on shielded conductors is noticeable weaker than magnetic field acting on unshielded conductors, magnetic field is concentrated into shielding. Therefore forces acting on shielded conductors are lower in comparison with forces acting on unshielded conductors. 


\section{ILUSTRATIVE EXAMPLE}

Input values

- The layout of the conductors is shown in Fig. 1. Thicknesses of the shielding jackets (two alternatives): $\delta=10 \mathrm{~mm}, \delta=5 \mathrm{~mm}$.

- Magnitude of the current densities in conductors: $J_{\text {ext }}=5 \cdot 10^{6} \mathrm{~A} / \mathrm{m}^{2}$

- Frequency: $f=50 \mathrm{~Hz}$

- Electrical conductivities in plane $(x, y)$ : conductors (conductor cables): $\gamma=0$ shielding jackets (electrical steel): $\gamma=6.67 \cdot 10^{6} \mathrm{~S} / \mathrm{m}$ other parts: $\gamma=0$

- Magnetic permeability:

magnetic permeability of shielding jackets is determined from the magnetization curve, Fig. 5

other parts: $\mu=\mu_{0}$.

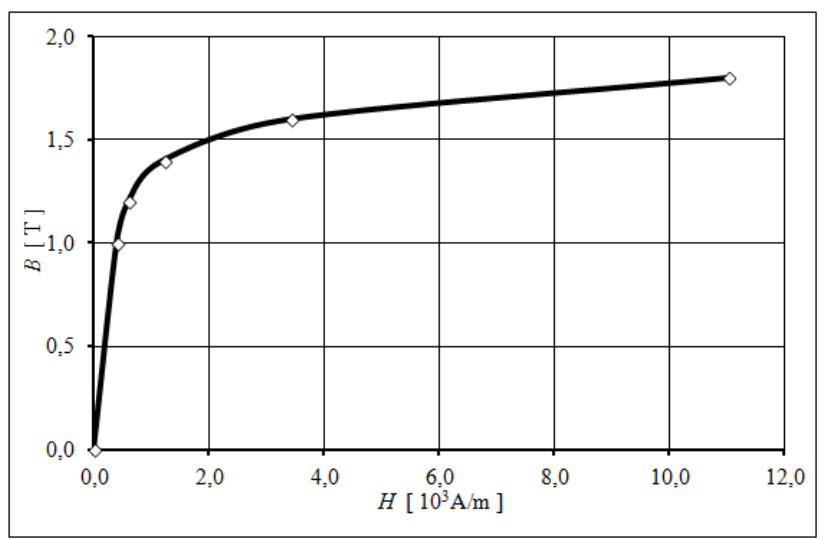

Fig. 5 Magnetization curve $B(H)$ of the electrical steel.

Results and discussion

Time function of the forces acting on unshielded and shielded conductors X, Y, Z can be seen in Figs. 6 to 8 .

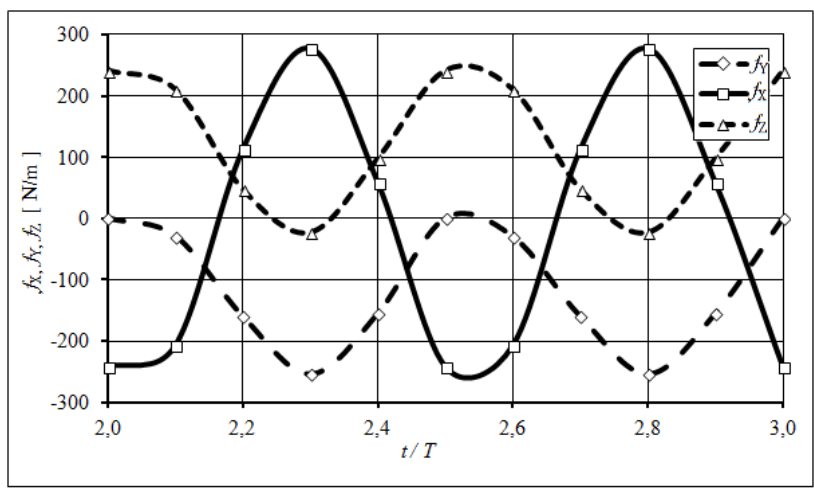

Fig. 6 Forces acting on the unshielded conductors.

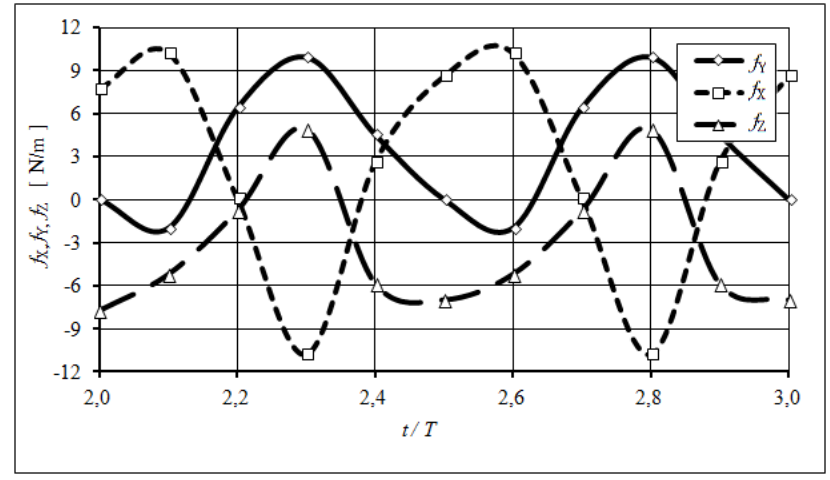

Fig. 7 Forces acting on the shielded conductors $(\delta=10 \mathrm{~mm})$.

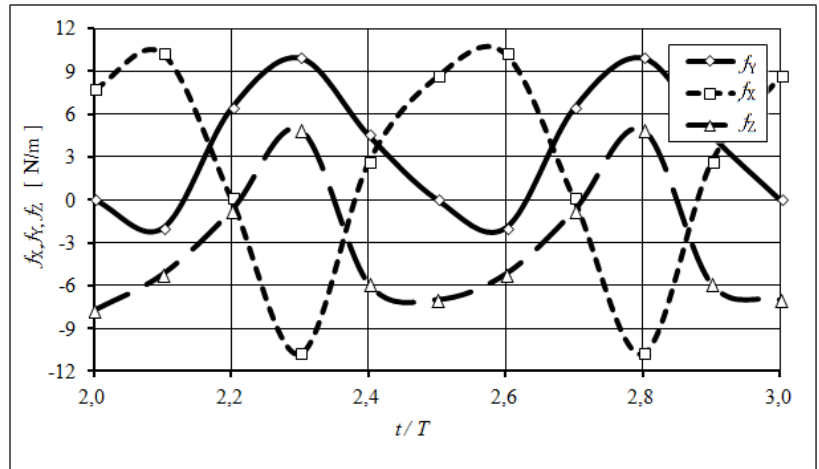

Fig. 8 Forces acting on the shielded conductors $(\delta=5 \mathrm{~mm})$.

Time function of the Joule losses density in shielding jackets can be seen in Fig. 9.

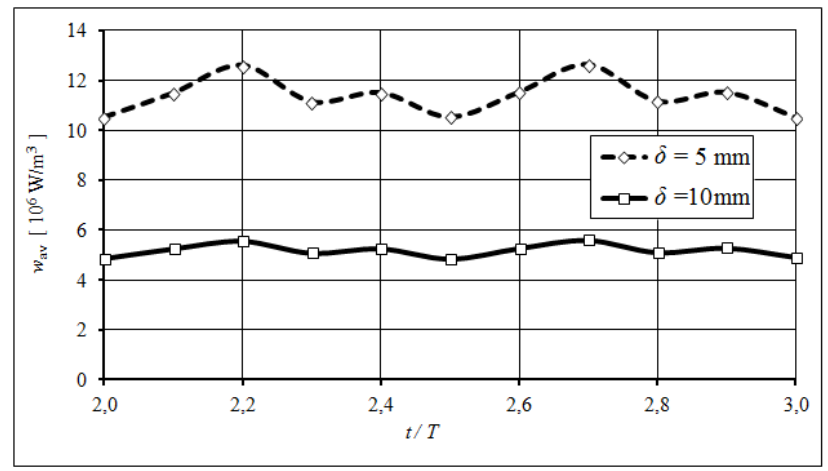

Fig. 9 Joule losses density in shielding jackets $(\delta=10 \mathrm{~mm}$, $\delta=5 \mathrm{~mm})$.

The calculation of forces acting on the conductors X, Y, $\mathrm{Z}$ shows, that only $x$ components of these forces has significant value, while calculated values of $y$ component are negligible and caused by numerical error. While forces acting on unshielded line achieve values of $280 \mathrm{~N} / \mathrm{m}$, forces acting on shielded line with the thickness of jacket $\delta=5 \mathrm{~mm}$ are reduced to value of mere $12 \mathrm{~N} / \mathrm{m}$ and to mere $10 \mathrm{~N} / \mathrm{m}$ while the thickness of the shielding jacket is $\delta=10 \mathrm{~mm}$. Similarly, values of $x$ and $y$ components of forces acting on 
the shielding jackets are negligible and under the value of numerical error.

Conductors affect each other by their magnetic field penetrating trough the shielding. This effect is the stronger, the thinner is the thickness of $\delta$. The mutual effect was negligible in considered examples of thicknesses $\delta=10 \mathrm{~mm}$ and $\delta=5 \mathrm{~mm}$.

\section{CONCLUSION}

It is necessary to deal with the forces acting on the supply lines for safe design of various power engineering devices. These can achieve high values. Presented paper proposes calculation of these forces for three phase input line arranged according to Fig.1. conducting currents of a symmetrical three phase system. To decrease the magnitude of forces, magnetic shielding of the conductors mad of steel pipes was designed. Based on results, it can be stated, that this method of shielding is very efficient.

However, considered method of shielding posses a disadvantage: eddy currents are induced into shielding jackets, these currents cause Joule losses and heating of shielding jackets. Solved example showed the density of these losses of approximately $\bar{w}_{\mathrm{av}}=1.1 \cdot 10^{7} \mathrm{~W} / \mathrm{m}^{3}(\delta=5 \mathrm{~mm})$ and $\bar{w}_{\mathrm{av}}=5 \cdot 10^{6} \mathrm{~W} / \mathrm{m}^{3}(\delta=10 \mathrm{~mm})$, while the current density in the conductors is $J_{\text {ext }}=5 \cdot 10^{6} \mathrm{~A} / \mathrm{m}^{2}$. This occurs considering both thicknesses $\delta$. This relatively low value od losses does not cause substantial heating of shielding jackets.

\section{ACKNOWLEDGEMENT}

The support of University of West Bohemia, research project SGS-2012-039, is gratefully acknowledged.

\section{REFERENCES}

[1] MAYER, D. - ULRYCH, B.: Numerical approach for computation of electromagnetic shielding. Journ. El. Eng., Vol. 64 (2013), No. 4, pp. 256-260.

[2] Quickfield. [online]. [cit. 2014-09-26]. Available via: http://quickfield.com

[3] WILSON, P.F. - MA, M.T.: Techniques for measuring the electromagnetic shielding effectiveness of materials. II. Near-field source simulation, Electromagnetic
Compatibility, IEEE Transactions on, vol.30, no.3, pp.251,259, doi: 10.1109/15.3303

[4] CELOZZI, S. - LOVAT, G. - ARANEO, R.: Electromagnetic Shielding. Hoboken. John Wiley and Sons, 2001. doi: 10.1109/15.3303

[5] MEMARI, A. R. - JANISCHEWSKYJ, W.: Mitigation of magnetic field near power lines, Power Delivery, IEEE Transactions on Power Delivery, vol.11, no.3, pp.1577-1586, 1996, doi: 10.1109/61.517519

Received December 11, 2014, accepted January 19, 2015

\section{BIOGRAPHY}

Daniel Mayer (1930), received the Ing., CSc. and DrSc. degrees in electrical engineering from Technical University in Prague, Czech Republic, in 1952, 1958 and 1979, respectively. In 1959 Associate Professor at the University of West Bohemia in Pilsen, in 1968 Full Professor of the Theory of Electrical Engineering. Research interests: circuit theory, electromagnetic field theory, electrical machines and apparatus, history of electrical engineering. He published 6 books, more than 320 scientific papers and 11 patents. He is a Fellow of the IET, member ICS, member of editorial boards of several international journals and leader of many grant projects

Bohuš Ulrych (1937), Assoc. Prof., CSc., has been working for a long time in the Department of Theory of Electrical Engineering at the Faculty of Electrical Engineering of UWB in Pilsen. His professional interests are aimed at modern numerical methods of solution of electromagnetic and coupled problems. Author and co-author of about 160 papers and several textbooks. Author of a lot of user's SW for calculation of electromagnetic fields and coupled problems.

Petr Polcar (1983). He graduated (Ing.) in 2007, in 2014 he defended his $\mathrm{PhD}$ thesis in the field of electromechanical systems using magnetic liquids. He works at the Department of Theory of Electrical Engineering at the University of West Bohemia. His scientific research aims on applied electromagnetic field theory with main focus on electromagnetic properites and applications of magnetic liquids. 